\title{
In Situ Electron Microscopy for Electrically Induced Charge Transport and Phase Transformation
}

\author{
Kai He${ }^{1 *}$
}

1. Department of Materials Science and Engineering, Clemson University, Clemson, USA

* Corresponding author: kaihe@clemson.edu

During the past decade, in situ transmission electron microscopy (TEM) has been recognized as one of the fast-growing techniques and attracted tremendous attention in a broad range of nanoscience research because of its unique capability to capture dynamic structural evolution and allow for mechanistic understanding of physical and chemical processes in various nanoscale materials systems [1]. Besides various in situ stimuli such as mechanical force, magnetic field, heat, etc., electric probes are more often used to trigger numerous electrically induced phenomena of materials and devices in different scenarios. Specifically, applying the electric field to chemically reactive environments can enable electrochemical reactions such as lithiation and sodiation of electrode materials for rechargeable ion batteries, which have been probed by the real-time TEM observations using dry-cell, liquid-cell, and MEMS-based setups [2]. Additionally, using electrical bias to energize functional devices can induce charge transport and lead to charge redistribution inside the key functioning components, such as the filamentary formation in resistive switching memories [3], and built-in potential in semiconductor heterojunctions [4]. All of these in situ experiments have been able to generate fruitful information and add new knowledge to understand the electrically induced phenomena and processes, which is also the main focus of our recent research.

Here we show our recent effort on investigation of the charge and mass transport induced and controlled by electrical bias in multiple functional materials and devices, where different combinations of in situ TEM imaging and analytical techniques are implemented according to specific needs, as illustrated in Figure 1. First, we aim to identify the phase transformation during electrochemical reactions under nonequilibrium conditions. Taking lithium ion battery as an example, previous studies have found different types of electrochemical reactions with lithium via various mechanisms such as intercalation, alloying, and conversion, which dictates the lithiation processes following the thermodynamic reaction pathways. However, in most realistic cases, these phase transitions proceed in distinct paths with each individual reactions overlapped in timescale. Therefore, we utilize in situ analytical TEM techniques to build direct correlations between microstructure and electrochemistry on atomic to nanoscale and reveal the kinetics of phase transformations during the electrochemical reactions. Figure 1a shows the in situ HRTEM images of atomically resolved structural evolution during lithiation of two-dimensional single-crystal $\mathrm{SnSe}_{2}$ nanoflakes and the corresponding electron diffraction confirming the associated phase transformation for reversible intercalation, conversion and alloying reactions throughout multiple electrochemical cycles. We have identified each intermediate phases and also elucidated that the reaction pathway and reversibility are strongly related to the kinetic effect in realistic non-equilibrium conditions [5].

We also make effort in understanding the origin of conductive channels and their growth dynamics in resistive switching memories, and we use in situ TEM to demonstrate in artificially engineered bilayer reduced graphene oxide films that nanoscale graphene oxide sheets bonded with oxygen dynamically change their physical and chemical structures upon an applied electric field. We clearly observe that a 
conductive graphitic channel with a conical shape evolves from the upper oxygen-rich region to the lower oxygen-poor region (Figure $1 \mathrm{~b}$ ), and such asymmetric oxygen distribution enables nonvolatile write-once-read-many memory behaviors without experiencing the bubble destruction due to the efficient migration of oxygen ions [3]. We are also interested in exploring fundamental questions such as how ions behave in response to electric stimulus, especially under extreme electric fields in vacuum. From a model study where $1000 \mathrm{~V}$ bias was applied to a pair of electrodes with separation of $10 \mu \mathrm{m}$ to form an ultrahigh electric field, we were able to observe the formation of electrospray of ionic liquid from the surface of the counter electrode, and then growth and solidification to form the dendritic structure (Figure 1c) [6]. Overall, the findings obtained from in situ TEM studies provide fundamental guidance for understanding the electrically induced charge transport and phase transformation that may not be easily accessible by other means [7].

References:

[1] H Zheng, YS Meng and Y Zhu, MRS Bulletin 40, 12 (2015).

[2] Y Yuan, K Amine, J Lu and R Shahbazian-Yassar, Nat. Commun. 8, 15806 (2017).

[3] S Kim et al., ACS Nano, 12, 7335 (2018),

[4] K He et al. Nanotechnology 24, 115703 (2013).

[5] S Kim et al. Adv. Mater. 30, 1804925 (2018).

[6] KJ Terhune, LB King, K He and J Cumings, Nanotechnology 27, 375701 (2016).

[7] The authors acknowledge the use of facilities at EM Facility of Clemson University, NUANCE-EPIC Center of Northwestern University, and CFN of Brookhaven National Laboratory.

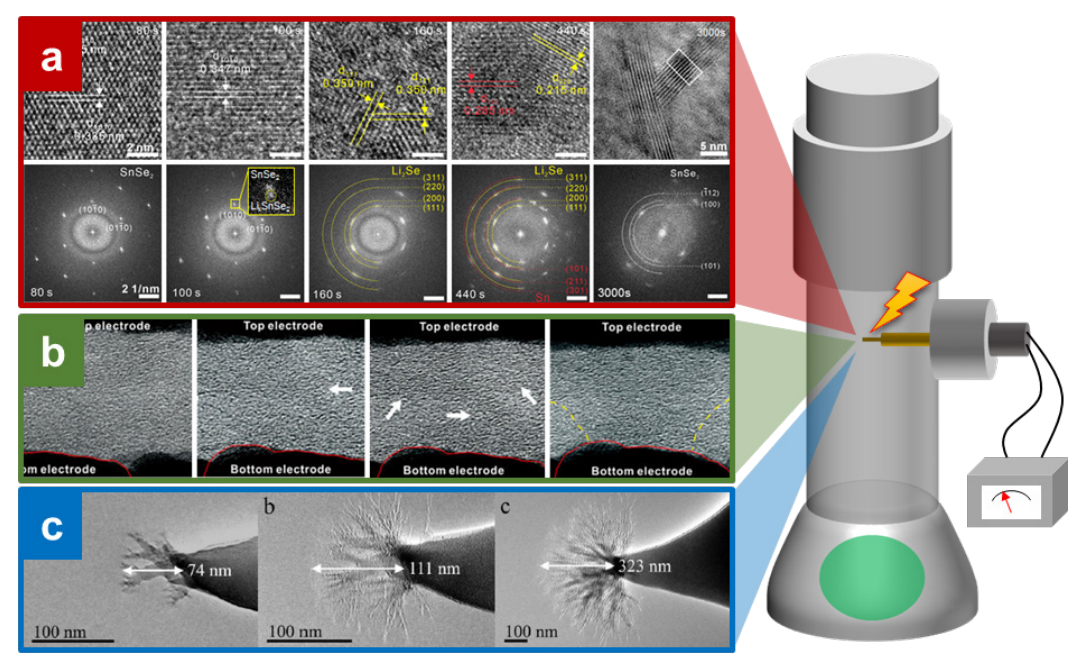

Figure 1. In situ TEM techniques enable real-time observation and analytical characterization for electrically induced materials phenomena in diverse research fields, such as (a) rechargeable batteries, (b) resistive switching memories, and (c) electrospray of ionic liquid. 